\title{
EGFR mutation and HPV infection in sinonasal inverted papilloma and squamous cell carcinoma*
}

\author{
Virginia N. Cabal', Marta Menéndez², Blanca Vivanco³, Sira Potes-Ares', \\ Cristina Riobello', Laura Suárez-Fernández', Rocío García-Marín', Verónica \\ Blanco-Lorenzo³, Fernando López², César Álvarez-Marcos², José Luis \\ Llorente'2, Mario A. Hermsen'
}

Rhinology 58: 4, 368 - 376, 2020

https://doi.org/10.4193/Rhin19.371

*Received for publication:

October 24, 2019

Accepted: January 23, 2020

'Dept Head and Neck Oncology, Instituto de Investigación Sanitaria del Principado de Asturias, Oviedo, Spain

2 Dept Otolaryngology, Hospital Universitario Central de Asturias, Oviedo, Spain

${ }^{3}$ Dept Pathology, Hospital Universitario Central de Asturias, Oviedo, Spain

\begin{abstract}
Background: To evaluate the involvement of EGFR signalling and HPV infection in a cohort of inverted sinonasal papilloma (ISP) and sinonasal squamous cell carcinoma (SNSCC) and their value for prognosis and clinical treatment.
\end{abstract}

Methods: We analysed 55 ISP, 14 SNSCC associated with ISP (SNSCC-isp) and and 60 SNSCC not associated with ISP (SNSCC-novo) for EGFR gene mutation and copy number gain, protein expression of EGFR and phosporylated EGFR (pEGFR), and HPV-infection and KRAS mutation. Findings were correlated to clinico-pathological and follow-up data.

Results: We found EGFR exon 20 mutations in 38\% (7/18) ISP, in 50\% (6/12) SNSCC-isp and in 5\% (1/19) SNSCC-novo. EGFR was expressed in 92\% of ISP, while pEGFR was observed in 54\% (21/39). SNSCC-isp and SNSCC-novo demonstrated comparable expression of EGFR ( $57 \%$ and 33\%) and of pEGFR (44\% and 38\%). We observed an inverse relation between EGFR exon 20 mutation and pEGFR expression. Four of 39 (10\%) ISP carried HPV-16. Oncogenic HPV was detected in 3/12 (25\%) SNSSC-isp and in 1/8 (13\%) SNSCC-novo. KRAS mutations were not detected in any of the samples. HPV infection was inversely correlated with pEGFR expression but not with EGFR mutation. ISP with EGFR activation by mutation or by phosphorylation had longer ISP-free survival, however, neither EGFR exon 20 mutation, pEGFR expression nor HPV infection demonstrated prognostic value in SNSCC.

Conclusions: EGFR exon 20 mutation is frequent in ISP and SNSCC-isp, while activation of EGFR through phosphorylation also plays an important role. Our data indicate that a large proportion of SNSCC patients could benefit from therapy with modern EGFR inhibitors.

Key words: sinonasal squamous cell carcinoma, inverted sinonasal papilloma, EGFR mutation, HPV infection, treatment

\section{Introduction}

Sinonasal squamous cell carcinoma (SNSCC) represents approximately $50 \%$ of all sinonasal malignancies. The incidence is less than 1 case per 100,000 inhabitants per year, occurring predominantly among men with a mean age of presentation of 50 to 60 years ${ }^{(1,2)}$. SNSCC occurs predominantly in the respiratory epithelium of the maxillary sinus and the nasal cavity and approximately $30 \%$ of cases are etiologically related to professional exposure to textile, leather, wood or aluminium ${ }^{(3,4)}$, whereas tobacco does not appear to play a key role. Histological changes that precede the development of SNSCC include squamous metaplasia and subsequent dysplasia ${ }^{(5)}$. In addition, inverted sinonasal papilloma (ISP) has been associated with an estimated $5-15 \%$ of of SNSCC ${ }^{(6,7)}$. Although distant and lymph node metastasis are exceptional, SNSCC carry a poor prognosis, with an overall 5 -year survival at $30-50 \%^{(1,2,8,9)}$ and new therapeutic options are needed.

Genetic data on these rare tumours are limited. Frequent TP53 mutation and $\mathrm{p} 53$ overexpression has been reported in up to $70 \%$ of SNSCC ${ }^{(10,11)}$. HPV infection has been described in $38 \%$ 
of benign and 31\% malignant tumours ${ }^{(6,7,12)}$. In addition, 21\% of SNSCC overexpressed p16INK4A (cyclin-dependent kinase inhibitor 2A), often used as surrogate marker of HPV-positive cases. However, the finding of many non-oncogenic or low-risk HPV subtypes made a clear role in malignant tranformation less likely ${ }^{(6,7,12,13)}$. Other indications for ISP as precursor to invasive SNSCC came from $X$ chromosome inactivation analysis, although in one case where both ISP and synchronous SNSCC could be analysed, the two samples showed $\mathrm{X}$ inactivation of different alleles, indicating that they arose from different clones ${ }^{(14)}$. Finally in 2015, Udager et al were the first to positively demonstrate a clonal relationship between ISP and synchronous and metachronous SNSCC by detecting identical EGFR mutations ${ }^{(15)}$. Moreover, they reported a high frequency of mutations, especially affecting EGFR exon 20 , in $88 \%$ of ISP and in $77 \%$ of SNSCC-isp). Two later studies confirmed the clonal EGFR mutations in pairs of ISP and SNSCC-isp, albeit in varying frequencies, from $30 \%$ up to $91 \%$ of tumours ${ }^{(16,17)}$.

It is possible that EGFR mutation and HPV infection represent two different pathways of progression from ISP to SNSCC. As the number of cases studied are still low, it remains uncertain whether EGFR mutation or HPV infection can predict the progression of ISP into SNSCC, or whether SNSCC with and without EGFR mutation or HPV infection carry a different prognosis.

Our aim was to study EGFR pathway activation and HPV infection in a series of ISP and SNSCC, and to correlate the findings to clinico-pathological characteristics and follow-up data. EGFR pathway activation was evaluated either through EGFR gene mutation, EGFR gene copy number gain, phosporylation of EGFR protein or, alternatively, by mutation of KRAS in the pathway downstream of EGFR itself.

\section{Material and methods}

\section{Samples and clinical data}

Our cohort is composed of 129 patients treated between 1989 and 2017 at the department of Otolaryngology, Hospital Universitario central de Asturias (Oviedo, Spain). Tumour samples were recollected retrospectively from the biobank archives of our hospital. All patients had signed an informed consent for the collection, analysis and storage of their biological material and the study was approved by the ethical committee (approval number $66 / 15$ for the project PI15/01629 and 07/16 for project CICPF16008HERM). All specimens were primary tumours, being 55 inverted papillomas (ISP), 14 SNSCC associated with ISP (SNSCC-isp) and 60 SNSCC without ISP association (SNSCCnovo). All SNSCC were of the keratinizing type. Treatment and follow-up data for all cases are described in Table 1.

Of 55 ISP patients, 19 were female (35\%) and 36 male (55\%).

Mean age was 60 years (range 27-81). According with the Krouse T Stage there were $18 \mathrm{~T} 1$ (33\%), 29 T2 (53\%) and 8 T3 (14\%).
None presented T4 stage. Eightteen originated in the maxillary sinus (33\%), 3 frontal sinus (5\%), 10 ethmoid (18\%) and 24 nasal cavity (44\%). Mean follow-up time was 141 months (range: 5-402). Recurrent disease in the form of a new ISP was observed during follow-up in 16 (29\%) cases. None of the 55 developed a SNSCC.

The SNSCC-isp group consisted of 6 metachronous and 8 synchronous cases. Nine were women (64\%) and 5 men (36\%). Mean age was 58 years (22-82 range). Eight tumours were located in the maxillary sinus (57\%), 4 ethmoid (29\%) and 2 nasal cavity (14\%). Two carcinomas were T2 (13\%), 4 T3 (29\%), 4 T4a (29\%) and 4 T4b (29\%). At the time of diagnosis, 3/14 (21\%) patients harboured lymph node metastasis. Mean follow-up time was 33 months (range: 3-159). During follow-up, 12/14 (86\%) developed recurrent SNSCC.

Among the 60 SNSCC-novo patients, 16 were female (26\%) and 44 male (74\%), with a mean age of 66 years (42-92 range). Forty-seven tumours were localized in maxillary sinus (78\%), 1 sphenoid sinus (2\%), 10 ethmoid (17\%) and 2 nasal cavity (3\%). One case was tumour stage T1 (2\%), 5 T2 (8\%), 16 T3 (27\%), 30 T4a (50\%) and 8 T4b (13\%), and 48 N0 (80\%), 10 N1 (17\%) and 2 N2 (3\%). At the time of diagnosis, 10 (21\%) patients harboured lymph node metastasis. Mean follow-up time was 28 months (range: 1-216). During follow-up, 48/60 (80\%) developed recurrent SNSCC.

\section{DNA extraction}

DNA was extracted from paraffin-embedded tissues with DNA QIAmp DNA Mini KIT (Qiagen GmbH, Hilden, Germany), according to the manufacturer's recommendations using an elaborate deparaffinization and lysis protocol published previously ${ }^{(18)}$. Normal blood DNA used as reference in the MLPA analysis was isolated with Roche High Pure Template Preparation Kit (Roche Diagnostics $\mathrm{GmbH}$, Manheim, Germany), following the supplier's guidelines.

\section{EGFR and KRAS mutation analysis}

Mutation in EGFR exon 20 and KRAS exon 2 (codons 12 and 13) were analysed by direct sequencing. While it is true that activating mutations may also occur in EGFR exons 18, 19 and 21, and in KRAS exon 3, the great majority occur in EGFR exon 20 and KRAS exon 2. Therefore, we decided to analyse only these regions. Amplification was carried out in a Simpliamp Thermal Cycler VXA24811 in standard conditions. PCR Primers and cycling conditions are detailed in Supplementary Table 1. PCR products were purified with Exo-BAP Mix (EURx Ltd, Gdansk, Poland) in accord with the manufacturer's specifications, and analysed by Sanger sequencing using the ABI PRISM 3100 and 3730 Genetic Analyser, (Applied Biosystems, Foster City, CA, USA). PCR conditions were as follows: EGFR exon 20 : $\left(5 \mathrm{~min} / 95^{\circ} \mathrm{C}\right.$ $+\left(15^{\prime \prime} 95^{\circ} \mathrm{C} / 30^{\prime \prime} 57^{\circ} \mathrm{C} / 1 \mathrm{~min} 72^{\circ} \mathrm{C}\right) \times 35$ cycles $+7 \mathrm{~min} / 72^{\circ} \mathrm{C}+$ 
Table 1. Clinical and follow-up data of all tumours.

\begin{tabular}{|c|c|c|c|c|}
\hline & & 55 ISP & 14 SNSCC-isp & 60 SNSCC-novo \\
\hline \multirow[t]{2}{*}{ Gender } & Female & 19/55(35\%) & $9 / 14(64 \%)$ & $16 / 60(26 \%)$ \\
\hline & Male & $36 / 55$ (65\%) & $5 / 14(36 \%)$ & $44 / 60(74 \%)$ \\
\hline \multirow[t]{2}{*}{ Age at Diagnosis } & Range & 27-81 years & $22-82$ years & $42-92$ years \\
\hline & Mean & 60 years & 58 years & 66 years \\
\hline \multirow[t]{3}{*}{ Differentiation } & Poor & n.a & $6 / 14(42 \%)$ & $25 / 60(42 \%)$ \\
\hline & Moderate & n.a & 4/14 (29\%) & $12 / 60(20 \%)$ \\
\hline & Well & n.a & 4/14 (29\%) & $23 / 60(38 \%)$ \\
\hline \multirow[t]{5}{*}{ T Stage } & $\mathrm{T} 1$ & n.a & $0(0 \%)$ & $1 / 60(2 \%)$ \\
\hline & $\mathrm{T} 2$ & n.a & 2/14 (13\%) & $5 / 60(8 \%)$ \\
\hline & T3 & n.a & 4/14 (29\%) & $16 / 60(27 \%)$ \\
\hline & $\mathrm{T} 4 \mathrm{a}$ & n.a & 4/14 (29\%) & $30 / 60(50 \%)$ \\
\hline & $\mathrm{T} 4 \mathrm{~b}$ & n.a & $4 / 14(29 \%)$ & $8 / 60(13 \%)$ \\
\hline \multirow[t]{3}{*}{ N Stage } & No & n.a & $11 / 14(79 \%)$ & $48 / 60(80 \%)$ \\
\hline & N1 & n.a & $3 / 14(21 \%)$ & $10 / 60(17 \%)$ \\
\hline & N2 & n.a & $0(0 \%)$ & $2(3 \%)$ \\
\hline \multirow[t]{4}{*}{ Krouse ISP T Stage } & $\mathrm{T} 1$ & 18/55 (33\%) & n.a & n.a \\
\hline & $\mathrm{T} 2$ & $29 / 55(53 \%)$ & n.a & n.a \\
\hline & T3 & $8 / 55$ (14\%) & n.a & n.a \\
\hline & $\mathrm{T} 4$ & $0(0 \%)$ & n.a & n.a \\
\hline \multirow[t]{5}{*}{ Localization } & Maxillary sinus & 18/55 (33\%) & $8 / 14(57 \%)$ & $47 / 60(78 \%)$ \\
\hline & Frontal sinus & $3 / 55(5 \%)$ & $0(0 \%)$ & $0(0 \%)$ \\
\hline & Sphenoid sinus & $0(0 \%)$ & $0(0 \%)$ & $1 / 60(2 \%)$ \\
\hline & Ethmoid & $10 / 55(18 \%)$ & 4/14 (29\%) & $10 / 60(17 \%)$ \\
\hline & Nasal cavity & $24 / 55$ (44\%) & $2 / 14(14 \%)$ & $2 / 60(3 \%)$ \\
\hline \multirow[t]{5}{*}{ Treatment } & None & $1 / 55(2 \%)$ & $0(0 \%)$ & $0(0 \%)$ \\
\hline & Surgery & $54 / 55$ (98\%) & 2/14 (14\%) & $15 / 60(25 \%)$ \\
\hline & Surgery+RT & $0(0 \%)$ & $11 / 14(79 \%)$ & $42 / 60(70 \%)$ \\
\hline & Surgery+CRT & $0(0 \%)$ & $1 / 14(7 \%)$ & $1 / 60(2 \%)$ \\
\hline & CRT & $0(0 \%)$ & $0(0 \%)$ & $2 / 60(3 \%)$ \\
\hline \multirow[t]{2}{*}{ Surgery } & Open & $6 / 54(11 \%)$ & $10 / 14(71 \%)$ & $57 / 58(98 \%)$ \\
\hline & Endoscopic & $48 / 54(89 \%)$ & 4/14 (29\%) & $1 / 58(2 \%)$ \\
\hline \multirow[t]{2}{*}{ Recurrence } & ISP & $16 / 55$ (29\%) & n.a & n.a \\
\hline & SNSCC & n.a & $12 / 14(86 \%)$ & $48 / 60(80 \%)$ \\
\hline Follow-up & Median & 115 months & 20 months & 15 months \\
\hline \multirow[t]{3}{*}{ Status } & NED & $42 / 55(76 \%)$ & $2 / 14(14 \%)$ & $5 / 60(8 \%)$ \\
\hline & DOD & $0 / 55(0 \%)$ & $12 / 14(86 \%)$ & $47 / 60$ (79\%) \\
\hline & DOC & $13 / 55(24 \%)$ & $0(\%)$ & $8 / 60(13 \%)$ \\
\hline
\end{tabular}

n.a.: not applicable, NED: no evidence of disease, DOD: died of disease, DOC: died of other causes.

$\left.4^{\circ} \mathrm{C}\right)$ and KRAS: $\left(5 \mathrm{~min} / 95^{\circ} \mathrm{C}+\left(30^{\prime \prime} 95^{\circ} \mathrm{C} / 45^{\prime \prime} 60^{\circ} \mathrm{C} / 1 \min 72^{\circ} \mathrm{C}\right) \mathrm{x}\right.$ 30 cycles $\left.+7 \mathrm{~min} / 72^{\circ} \mathrm{C}+4^{\circ} \mathrm{C}\right)$.

\section{Immunohistochemistry}

Immunohistochemistry (IHC) was performed on an automatic staining workstation (Dako Autostainer Plus; DakoCytomation).
The antibodies used for IHC were: anti-EGFR clone 2-18C9 (DAKO, Glostrup, Denmark) and anti-pEGFR clone D7A5 (Cell Signaling Technology, Cambridge, UK). The immunostaining was evaluated by two experienced investigators (MM and BV). EGFR and pEGFR immunostaining was considered positive when moderate to strong membranous, cytoplasmic, or both staining 
Table 2. Genotype of the EGFR exon 20 mutations and HPV status.

\begin{tabular}{|c|c|c|c|c|}
\hline Patient & Tumour Type & Mutation & Described before & HPV infection \\
\hline 8 & SNSCC-isp Synchronous & p.S778_D770 dup SVD / c.2303_2311 dup GTGTGGACA & ref 15,21 & Negative \\
\hline 69 & SNSCC-isp Synchronous & p.D770_N771 ins G / c.2310_2311 ins GGT & ref 15,21 & Negative \\
\hline 77 & SNSCC-isp Synchronous & p.D770_N771 ins GL / c.2310_2311 ins GGGTTA & ref 15,21 & HPV 16 \\
\hline 126 & ISP & p.D770_N771 ins SVD / c.2311_2312 ins GCGTGGACA & ref 21 & n.d. \\
\hline 33 & ISP & p.D770_N771 ins SVD / c.2311_2312 ins GCGTGGACA & ref 21 & n.d. \\
\hline 18 & ISP & p.D770_N771 ins SVD / c.2311_2312 ins GCGTGGACA & ref 21 & Negative \\
\hline 16 & ISP & p.N771 delins GS / c.2310_2311 AA > GGGTC & NO & n.d. \\
\hline 5 & ISP and SNSCC-isp Metachronous & p.N771 delins GY / c.2310_2311 A>GGGT & ref 15 & Negative \\
\hline 4 & SNSCC-isp Synchronous & p.N771 delins GY / c.2310_2311 A>GGGT & ref 15 & HPV 42 \\
\hline 22 & ISP & p.N771_P772 ins V / c.2313_2314 ins GTC & ref 15 & Negative \\
\hline 76 & SNSCC-novo & p.P772_H773 dup PDN / c.2315_2323 dup GGACAACCC & NO & HPV 16 \\
\hline 6 & SNSCC-isp Metachronous & p.H773_V774 ins NPH / c.2319_2320 ins AACCCCCAC & ref 21 & HPV 16 \\
\hline 30 & ISP & p.H773_V774 ins NPH / c.2319_2320 ins AACCCCCAC & ref 21 & HPV 16 \\
\hline 31 & ISP & p.H773_V774 ins NPH / c.2319_2320 ins AACCCCCAC & ref 21 & n.d. \\
\hline
\end{tabular}

ref: reference; n.d.: no data.

was observed in at least $10 \%$ of tumour cells; tumours with no or weak staining were regarded as negative.

\section{Multiplex Ligation-dependent probe amplification (MLPA)} MLPA was performed as described in detail previously ${ }^{(19)}$ using SALSA P105D-2 Glioma-2 (MRC-Holland, Amsterdam, The Netherlands) that contains 56 probes (including 10 for reference and 4 as internal control). This mix allows the detection of aberrant copy number of EGFR using 11 specific probes. Relative copy number values, including standard deviation, higher than 1.25 were considered as gains and 2.0 or higher as amplifications.

\section{HPV DNA detection}

The quality of the extracted DNA was checked by PCR amplification of $\beta$-globin (forward primer 5'-ACACAACTTGTGTGTTCACTAGC-3' and reverse primer 5'-CAAACTTCATCCACGTTCACC-3'). PCR with MY11/GP6+ primers (site-directed L1 fragment of HPV) was performed in order to detect a broad spectrum of HPV genotypes ${ }^{(11,20)}$. Briefly, the PCR was performed in $25 \mu \mathrm{l}$ of reaction mixture containing $1 \times$ PCR buffer, $2 \mathrm{mmol} / \mathrm{L}$ $\mathrm{MgCl}_{2}, 50 \mu \mathrm{mol} / \mathrm{L}$ of each deoxynucleoside, $0.5 \mu \mathrm{mol} / \mathrm{L}$ of sense and antisense primers, $10 \mu \mathrm{l}$ of DNA sample and $1 \mathrm{U}$ Taq DNA polymerase (Promega Biotech Iberica S.L. Madrid, Spain), by thermal profile of 35 cycles: denaturation at $94^{\circ} \mathrm{C}$ for $30 \mathrm{sec}$, annealing at $55^{\circ} \mathrm{C}$ for $30 \mathrm{sec}$ and extension at $72^{\circ} \mathrm{C}$ for $1 \mathrm{~min}$, with an initial denaturation at $94^{\circ} \mathrm{C}$ for $5 \mathrm{~min}$ and a final extension at $72^{\circ} \mathrm{C}$ for $10 \mathrm{~min}$. The amplified DNA fragments of approximately 200 bp were identified by electrophoresis in $1.5 \%$ agarose gel with ethidium bromide. All positive specimens for L1 fragment were tested by hybridization assays using type-specific probes for HPV (Supplementary Table 1).

\section{Statistical analysis}

SPSS 12.0 software (SPSS Inc., Chicago, IL, USA) was used to analyse correlations existent between clinical and genetic factors using Pearson chi-square and Fisher tests. Continuous variables were analysed with Student's t test. Kaplan-Meier curves were undertaken for the evaluation of survival by application of logarithmic range test (Log-range test) considering significance for values under $\mathrm{p}=0.05$.

\section{Results}

Genetic alterations in EGFR, KRAS, and HPV detection DNA was extracted from all 129 samples included in this study. Unfortunately, interpretable EGFR exon 20 sequences were only obtained in 49 cases due to the bad quality and low quantity of DNA obtained from paraffin-embedded tumour material. In total 14 samples, 7/18 (39\%) ISP, 6/12 (50\%) SNSCC-isp and 1/19 (5\%) SNSCC-novo, carried mutations in EGFR (Supplementary Table 2). All mutations exclusively concerned in-frame insertions affecting the region S768-V774 in exon 20 (Table 2). To our knowledge, two mutations had not been described before in the literature on sinonasal tumours (Supplementary Figure 1). EGFR gene copy number gain as evaluated by MLPA was very infrequent, observed in only $1 / 17$ (6\%) ISP, $1 / 5$ (20\%) SNSCC-isp and 6/32 (19\%) of SNSCC-novo (Supplementary Table 2). EGFR protein expression was observed in 34/37 (92\%) ISP, 6/10(60\%) SNSCC-isp and in 24/54 (44)\% SNSCC-novo, while EGFR activation by phosphorylation (pEGFR) was observed in 21/39 

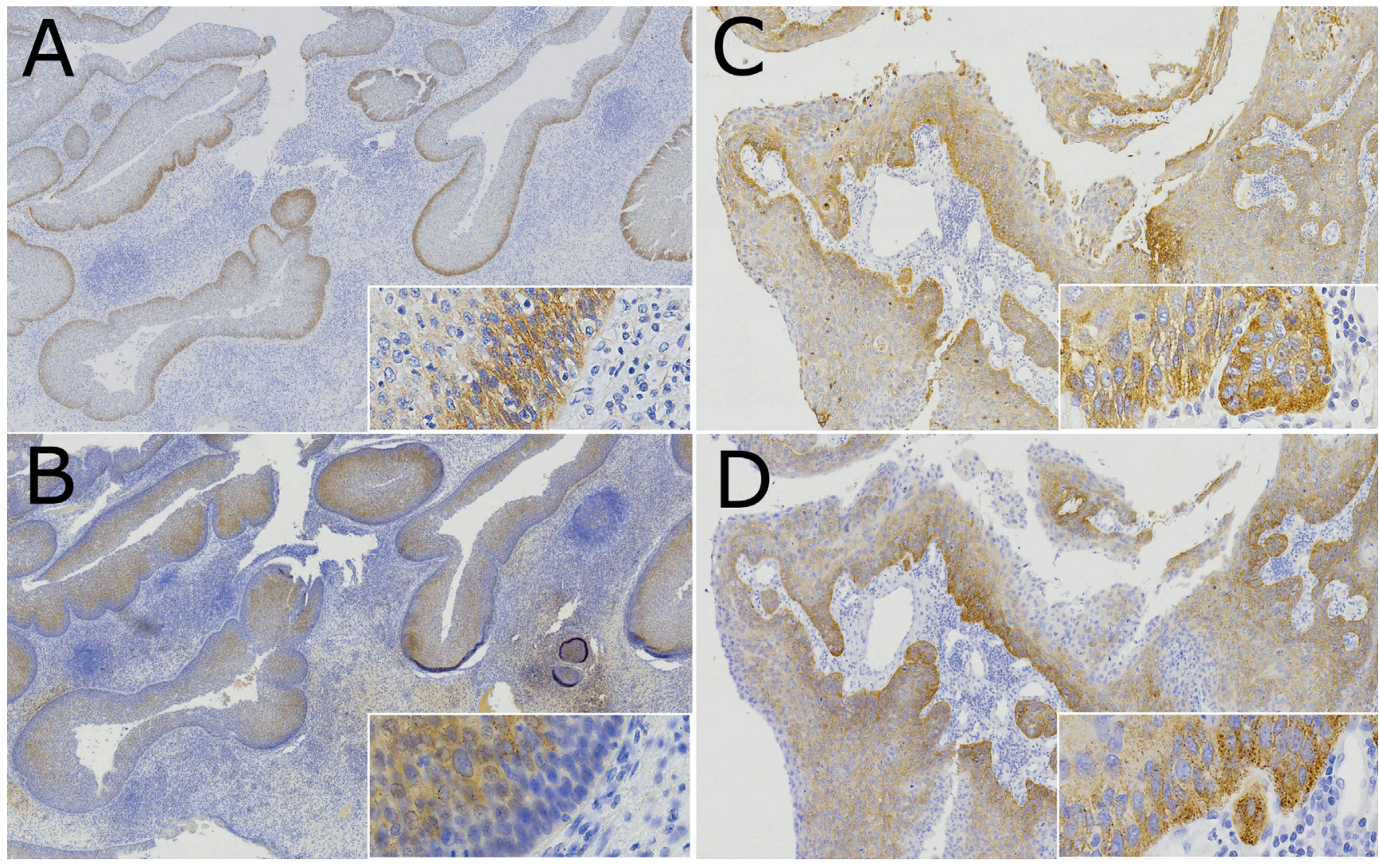

Figure 1. Photomicrographs of immunohistochemical expression of EGFR ( $A$ and $C$ ) and pEGFR ( $B$ and $D)$ detected in the cytoplasm and the cell membrane of an ISP ( $A$ and B; original magnification 4x and insert 40x) and a SNSCC-novo (C and D; original magnification 10x and insert 40x).

(54\%) ISP, 4/12 (33\%) SNSCC-isp and 20/52 (38\%) SNSCC-novo (Figure 1). Four of 40 (10\%) ISP samples harboured HPV-16 and no low-risk HPV types were detected. Among the 13 analysed SNSCC-isp, two cases showed presence of HPV type 16, one type 18 and two low-risk types 42 and 61. In contrast, only 1/50 (2\%) SNSCC-novo was found positive with HPV type 16 (Supplementary Table 2 and Figure 2). No KRAS mutations were detected in all of the analysed ISP, SNSCC-isp and SNSCC-novo samples (Supplementary Table 2).

\section{Correlations between genetic alterations and clinical data} Univariate analysis of clinical and genetic characteristics of 14 SNSCC-isp and 60 SNSCC-novo showed that the former occurred more frequently in women (64\% versus $26 \%$, Fisher exact $\mathrm{Chi}^{2}, \mathrm{p}=0.012$ ), at a younger age of onset (58 years versus 66 years, Student's t-test, $\mathrm{p}=0.031$ ) and more often (though not significantly) in the ethmoid sinus than SNSCC de novo ( $29 \%$ versus 15\%). There were no significant differences in T stage, $\mathrm{N}$-stage, degree of differentiation, nor in clinical treatment or clinical course (Table 1). EGFR exon 20 mutations were found significantly more frequently in SNSCC-isp than SNSCC-novo, respectively 6/12 (50\%) versus $1 / 19$ (5\%) (Fisher exact $\mathrm{Chi}^{2}$, $\mathrm{p}=0.007$ ), whereas EGFR and pEGFR expression and also EGFR copy number gains were similar in the two groups of tumours.
HPV infection (all subtypes) occurred significantly more frequent in SNSCC-isp than in SNSCC-novo, respectively 5/13 (38\%) versus $1 / 50$ (2\%) (Fisher exact $\mathrm{Chi}^{2}, \mathrm{p}=0.001$ ). This difference was also significant considering only the oncogenic subtypes, respectively $3 / 13$ (38\%) versus $1 / 50$ (2\%) (Fisher exact $\mathrm{Chi}^{2}, \mathrm{p}=0.025$ ). Multivariate logistic regression analysis (Table 3 ) of the four variables that were significantly different in univariate analysis showed only EGFR mutation as an independent significant factor differentiating SNSCC-isp and SNSCC-novo (adjusted odds ratio $25.953, \mathrm{p}=0.033$ ).

Analysing all 74 SNSCC, we found no correlation between EGFR mutation and T stage, $\mathrm{N}$ stage, grade of differentiation, tumour localization, age, or overall, disease-specific or disease-free survival. Also EGFR or pEGFR expression and HPV infection were not related to any of the clinical parameters or survival. Similarly among the 55 ISP, there was no relation between EGFR mutation, EGFR or pEGFR expression or HPV infection and Krouse T stage, tumour localization, age, or gender. However, Kaplan Meier analysis showed longer ISP-free survival in cases with EGFR pathway activation, either by mutation or by phosphorylation (Log rank 3.636, $\mathrm{p}=0.057$ ) (Figure 3A). Longer ISP-free survival was also observed in cases with HPV infection (Log rank 1.043, $\mathrm{p}=0.307$ ) (Figure 3B).

Finally, including all 55 ISP and all 74 SNSCC cases, we analysed 


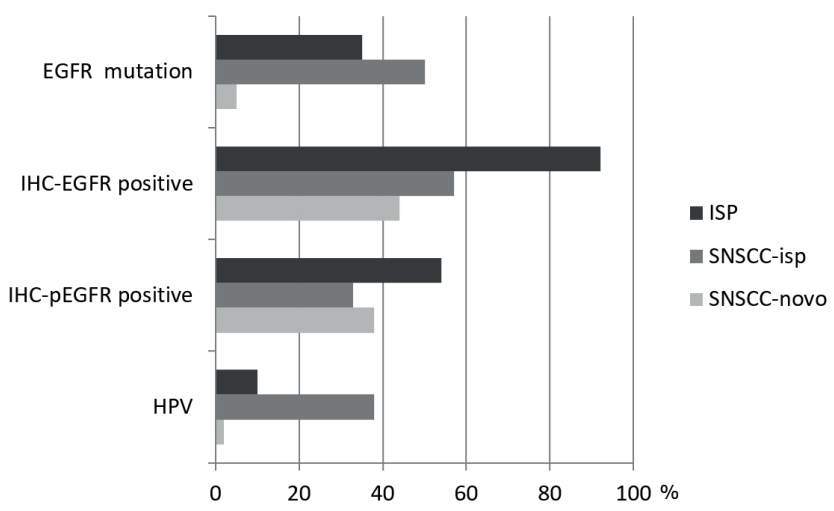

Figure 2. Graphic representation of the frequencies of EGFR mutation, EGFR and pEGFR protein expression, and HPV infection according to type of tumour sample.

possible relations between the genetic data. We observed an inverse (although not completely exclusive) relation between EGFR mutation and pEGFR expression, with 3/12 (25\%) EGFR mutated cases versus 18/27 (67\%) EGFR wildtype cases also expressing pEGFR (Fisher exact $\mathrm{Chi}^{2}, \mathrm{p}=0.035$ ). Also the presence of oncogenic HPV occurred exclusively in cases with no pEGFR expression (0/4 (0\%) HPV-positive cases versus 34/82 (67\%) HPVnegative cases expressing pEGFR, Fisher exact Chi2, $\mathrm{p}=0.149$ ). However, we found oncogenic HPV-positivity co-occurring with EGFR mutation in two ISP and also two SNSCC-isp (Table 2 and Supplementary Figure 2).

\section{Discussion}

In the present study we found EGFR exon 20 insertion mutations in an elevated proportion of ISP and SNSCC-isp, but not in SNSCC-novo, confirming previous studies ${ }^{(15-17,21)}$. However, in contrast to these studies, HPV infection was infrequent and did not occur mutually exclusive with EGFR mutation. In addition, we found few cases with EGFR copy number gains, however, activated pEGFR expression occurred frequent in ISP and SNSCCisp and does appear mutually exclusive with EGFR mutation. The number of cases SNSCC-isp in this study is relatively small, however, it must be taken into account that SNSCC is a rare tumour and, within this group of tumours, those associated with inverted papillomas are even less frequent. Comparable series in the literature included $17-23$ cases $^{(15-17)}$. The clinical features of our cohort of ISP and SNSCC were similar, to those published in previous studies ${ }^{(15-17,21)}$. Our data confirm the earlier age of onset of SNSCC-ISP compared to SNSCC-novo $(p=0.031)^{(16,22)}$. We also found a higher proportion of women in the SNSCC-isp group (64\%) as opposed to the other groups, however, this was not observed in other studies. The frequency of EGFR mutations in ISP (39\%) was notably less than in previous studies that reported $72-91 \%$ of mutated tumours ${ }^{(15-17,21)}$. Among the SNSCC-isp this was different; we identified $50 \%$ of tumours with mutation,
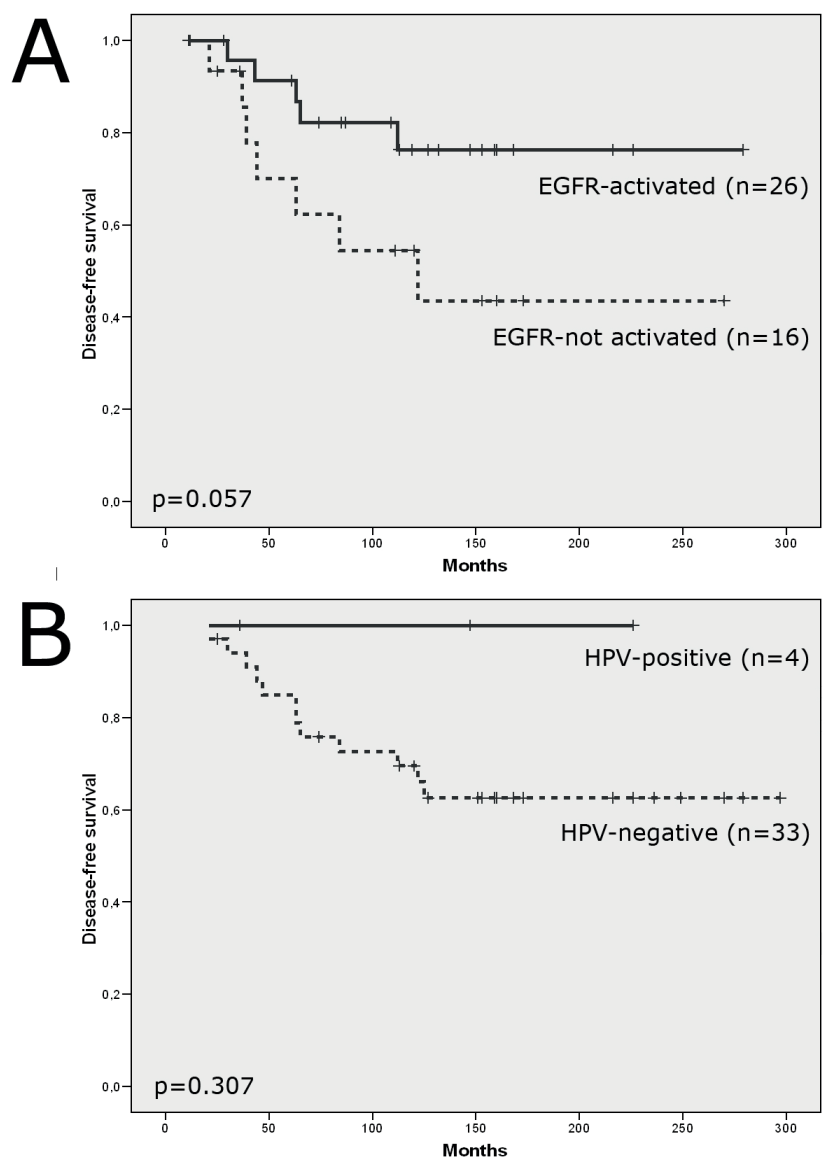

Figure 3. A) Disease-free survival of inverted sinonasal papillomas, 26 with and 16 without EGFR activation. B) Disease-free survival of inverted sinonasal papillomas, 4 with and 33 without HPV infection.

while a Japanese study found $30 \%^{(17)}$ and American and Italian studies respectively $77 \%$ and $72 \%(16,22)$. We have no explanation for these differences. Sasaki et al. ${ }^{(17)}$ investigated possible geographic or ethnic factors, also taking into consideration frequencies of EGFR mutation in lung cancer, but concluded that there is no evidence for that.

Protein expression of EGFR and the activated form pEGFR was not studied before in ISP or SNSCC-isp. Our data showed that 92\% ISP, 60\% SNSCC-isp and 44\% SNSCC-novo expressed EGFR. A proportion of these tumours also showed pEGFR positivity. Interestingly, the activation of EGFR by mutation and by phosphorylation appeared inversely correlated, suggesting that the EGFR pathway plays an important role both in SNSCC-isp and SNSCC-novo, although activation of the pathway may occur through different mechanisms.

Wild-type EGFR needs binding of its ligand for homo- or heterodimerization and subsequent autophosphorylation to become activated and regulate cell migration, proliferation and survival. EGFR mutations in exon 19 and L858R can cause constitutive dimerization and autophosphorylation without the need of ligand binding ${ }^{(23)}$, however, exon 20 insertion mutations lead to a pro- 
Table 3. Multivariate logistic regression analysis of variables in SNSCC-isp and SNSCC-novo.

\begin{tabular}{lcccccc} 
& & \multicolumn{2}{c}{ Univariate } & \multicolumn{2}{c}{ Multivariate } \\
& SNSCC-isp & SNSCC-novo & Statistical test & Significance & $\begin{array}{c}\text { Adjusted odds } \\
\text { ratio }\end{array}$ & Significance \\
\hline EGFR-mutation & $6 / 12(50 \%)$ & $1 / 19(5 \%)$ & Fisher Exact Chi & $\mathrm{p}=0.007$ & 25.953 & $\mathrm{p}=0.033$ \\
Gender (female/male) & $9 / 5$ & $16 / 44$ & Fisher Exact Chi & $\mathrm{p}=0.012$ & 12.847 & $\mathrm{p}=0.057$ \\
Age (years) & 58 & 66 & Student t-test & $\mathrm{p}=0.031$ & 1.016 & $\mathrm{p}=0.641$ \\
HPV-infection & $5 / 13(38 \%)$ & $1 / 50(2 \%)$ & Fisher Exact Chi ${ }^{2}$ & $\mathrm{p}=0.001$ & 0.533 & $\mathrm{p}=0.650$
\end{tabular}

tein conformation change that activates signalling independent of dimerization and autophosphorylation ${ }^{(24)}$. This may explain our finding that EGFR exon 20 mutation and expression of phosphorylated EGFR occur in a mutually exclusive manner. We also found that EGFR gene copy number gain never co-occurred with EGFR mutation, while it was more frequent in cases with EGFR and pEGFR immunopositivity, suggesting that gains partly may be responsible for the elevated expression.

HPV infection has long time been considered as an important oncogenic factor in SNSCC ${ }^{(7,12-13)}$. High risk HPV subtypes have been related with SNSCC-novo, while lower risk subtypes would be more frequent in ISP or SNSCC-isp ${ }^{(16,22)}$. Moreover, the existence of HPV infection and EGFR mutation was considered almost exclusive ${ }^{(22)}$. The results from our cohort are not in agreement with these studies. We found that 100\% (4 of 4) ISP and $60 \%$ (3 of 5) SNSCC-isp to be HPV-positive with subtypes 16 or 18, while 1 SNSCC-novo case harboured HPV type 16. In fact, only two samples were found with low-risk HPV (subtypes 42 and 61) and these were SNSCC-isp. In addition, we did not find that HPV infection and EGFR mutation were mutually exclusive. In four samples (two SNSCC-isp and two ISP), we detected simultaneous HPV-16 and EGFR exon 20 mutation. We believe that a role for oncogenic HPV in the tumourigenesis of SNSCC, either through premalignant ISP or in SNSCC-novo, still remains to be demonstrated.

The presence of EGFR mutation in ISP has been correlated with a lower risk of developing SNSCC ${ }^{(16,22)}$. Although our study did not include ISP samples of patients that later developed SNSCC, we did find that cases with EGFR pathway activation (by mutation or phosphorylation) carried lower risk of developing recurrent ISPs (Figure 3A). Somehow in contrast with these data, Sasaki et al. ${ }^{(17)}$ observed a worse 5-year overall survival of SNSCC carrying EGFR mutation compared to EGFR wildtype (although not significant, $48 \%$ versus $65 \%$, respectively). In addition, Takahashi et al. ${ }^{(25)}$ found correlation between EGFR protein expression and worse overall and disease-free survival in 67 SNSCC. However, in a similar study on 54 SNSCC, Lopez et al found no such correlation ${ }^{(19)}$. In our present study we found neither worse overall nor worse disease-free survival for SNSCC with EGFR mutation or EGFR activation through phosporylation. This may in part be explained by the 5 -year disease-specific survival of $23 \%$ in our series of 74 SNSCC, which is low compared to the literature reporting an average of $50 \%$. Indeed our cohort contains a high proportion (62\%) of stage T4a/T4b tumours and a recurrence rate of $81 \%$ (Table 1). This possibly reflects the fact that our hospital is a center of referral for sinonasal and skull base tumours, therefore receiving a relatively high number of complicated cases. The majority of patients were treated with open surgical approaches. Although endoscopic surgery has become common for sinonasal tumours, in most SNSCC an open approach is more appropriate, particularly when the tumour has infiltrated the hard palate, external maxillary wall, orbital fat or extraocular muscles, orbital apex, brain, or facial soft tissue. HPV-positive ISP have been reported to have a significantly higher risk of progression to SNSCC ${ }^{(22)}$. Our data only allowed to evaluate the risk of ISP to develop recurrent ISP and showed oncogenic HPV infection only in 4 cases with no recurring ISP (Figure 3B). This again demonstrates that the role of HPV in ISP, SNSCC-isp and SNSCC-novo still need further study.

Apart from a possible prognostic value of EGFR exon 20 mutations, the fact that an elevated percentage of ISP and SNSCC-isp carry these mutations opens up the possibility for therapy with specific inhibitors. All mutations found in our series, including the two novel mutations not described before in the literature on sinonasal tumours (Table 2, Supplementary figure 1), were situated exclusively in the $\mathrm{S768-V774}$ region of the protein kinase region of EGFR (enclosing codons 18 to 24 ) promoting a more active state that triggers the pathway in a ligand- and autophosphorylation-independent manner. EGFR exon 20 insertion mutation inhibitors as neratinib, dacomitinib, afitinib specifically targeting this region have yielded only minimal responses(23). Osirmitinib, a third generation EGFR exon 20 inhibitor has shown more promising results and has recently been approved as first-line therapy in NSCLC(26). As new exon 20 insertionselective inhibitors are being developed and preclinically tested, at the present moment perhaps the most promising compound being tested in an ongoing phase II clinical trial is poziotinib(27), which may also be effective against exon 20 insertion mutations in ERBB2. To date, there are no data on the usefulness of these inhibitors in SNSCC. Using an EGFR exon 20 mutated SNSCC 
tumour cell lines as model, Udager et al did show that second generation inhibitors, especially neratinib, caused strong growth inhibition, as opposed to first generation inhibitors ${ }^{(15)}$. This finding demonstrates the potential usefulness of treatment for patients with SNSCC. While KRAS and BRAF mutations could be negative response predictors for anti-EGFR therapies, fortunately our study and also previous studies have shown that neither ISP nor SNSCC-isp or SNSCC-novo carry these mutations ${ }^{(28,29)}$.

\section{Conclusion}

In conclusion, we found EGFR exon 20 insertion mutations in $39 \%$ ISP and 50\% SNSCC-isp. Protein expression of the activated form of EGFR was also observed frequently in these tumours as well as in SNSCC-novo, while HPV infection does not appear to play a critical role. As none of these tumours carry KRAS mutation, these results show that a large proportion of patients with SNSCC could be treated with EGFR antagonists, either irreversible small molecule inhibitors targeting exon 20 mutation in SNSCC-isp, monoclonal antibodies anti-EGFR in SNSCC-novo, or alternatively, with inhibitors downstream in the EGFR signalling pathway.

\section{Acknowledgements}

The authors thank Santiago Melón for the aid with the HPV analysis, and the technicians Eva Allonca and Aitana Vallina for retrieving and processing the paraffin embedded tissue samples and part of the immunohistochemical stainings. This study was supported by grants PI15-1629 from the Fondos de Investigación Sanitaria (FIS), grant CICPF16008HERM of Fundación AECC, and grant CB16/12/00390 from the Centro de Investigación Biomédica en Red de Cancer (CIBERONC), Spain. Plan Nacional de I+D+I 2013-2016 of the Plan Estatal cofinanced by the FEDER Funding Program from the European Union.

\section{Authorship contribution}

VNC, MM, JLL, CAM and MAH contributed to the study conception and design. MM, FL, CAM and JLL were responsible for tissue sample and clinical data collection. Material preparation, data collection and analysis were performed by VNC, MM, BV, SPA, LSF, CR, RGM and VBL. The first draft of the manuscript was written by VNC and MAH and all authors commented on previous versions of the manuscript. All authors read and approved the final manuscript.

\section{Conflict of interest}

The authors declare that they have no conflict of interest.

\section{References}

1. Ansa B, Goodman M, Ward K et al. Paranasal sinus squamous cell carcinoma incidence and survival based on Surveillance, Epidemiology, and End Results data, 1973 to 2009. Cancer 2013; 119:2602-2610.

2. Turner JH, Reh DD. Incidence and survival in patients with sinonasal cancer: a historical analysis of population-based data. Head Neck 2012; 34:877-885.

3. Bonzini M, Battaglia P, Parassoni D et al. Prevalence of occupational hazards in patients with different types of epithelial sinonasal cancers. Rhinology 2013; 51:31-36.

4. Sanghvi S, Khan MN, Patel NR, Yeldandi S, Baredes S, Eloy JA. Epidemiology of sinonasal squamous cell carcinoma: A comprehensive analysis of 4994 patients. Laryngoscope 2014; 124:76-83.

5. El-Naggar AK, Chan J, Takata T, Grandis J, Slootweg P. WHO Classification of Tumours Pathology and Genetics of Head and Neck Tumours. Vol 4th ed. IARC Press, Lyon; 2017.

6. Alos L, Moyano S, Nadal A et al. Human papillomaviruses are identified in a subgroup of sinonasal squamous cell carcinomas with favorable outcome. Cancer 2009; 115:2701-2709.

7. Bishop JA, Guo TW, Smith DF et al. Human papillomavirus-related carcinomas of the sinonasal tract. Am J Surg Pathol 2013; 37:185-192.

8. Youlden DR, Cramb SM, Peters S et al.
International comparisons of the incidence and mortality of sinonasal cancer. Cancer Epidemiol 2013; 37:770-779.

9. Llorente JL, López F, Suárez C, Hermsen MA Sinonasal carcinoma: clinical, pathological, genetic and therapeutic advances. Nat Rev Clin Oncol 2014; 11:460-472.

10. Holmila R, Bornholdt J, Heikkilä P et al. Mutations in TP53 tumour suppressor gene in wood dust-related sinonasal cancer. Int J Cancer 2010; 127:578-588

11. García-Inclán C, López-Hernández A, Alonso-Guervós $M$ et al. Establishment and genetic characterization of six unique tumour cell lines as preclinical models for sinonasal squamous cell carcinoma. Sci Rep 2014; 4:4925.

12. Syrjänen $K$, Syrjänen S. Detection of human papillomavirus in sinonasal carcinoma: systematic review and meta-analysis. Hum Pathol 2013; 44:983-991.

13. Larque $A B$, Hakim S, Ordi J et al. High-risk human papillomavirus is transcriptionally active in a subset of sinonasal squamous cell carcinomas. Mod Pathol 2014; 27:343351.

14. Califano J, Koch W, Sidransky D, Westra WH. Inverted sinonasal papilloma : a molecular genetic appraisal of its putative status as a precursor to squamous cell carcinoma. Am J Pathol 2000; 156:333-337.

15. Udager AM, Rolland DCM, MCHugh JB, et al. High-Frequency Targetable EGFR Mutations in Sinonasal Squamous Cell Carcinomas Arising from Inverted Sinonasal Papilloma. Cancer Res 2015; 75:2600-2006.

16. Sahnane N, Ottini G, Turri-Zanoni M et al. Comprehensive analysis of HPV infection, EGFR exon 20 mutations and LINE1 hypomethylation as risk factors for malignant transformation of sinonasal-inverted papilloma to squamous cell carcinoma. Int J cancer 2019; 144:1313-1320.

17. Sasaki E, Nishikawa D, Hanai N, Hasegawa Y, Yatabe Y. Sinonasal squamous cell carcinoma and EGFR mutations: a molecular footprint of a benign lesion. Histopathology 2018; 73:953-962.

18. López F, Llorente JL, García-Inclán C et al. Genomic profiling of sinonasal squamous cell carcinoma. Head Neck 2011; 33:145153.

19. López F, Llorente JL, Oviedo CM et al. Gene amplification and protein overexpression of EGFR and ERBB2 in sinonasal squamous cell carcinoma. Cancer 2012; 118:1818-1826.

20. Álvarez-Argüelles ME, Melón S, Junquera $\mathrm{ML}$ et al. Human papillomavirus infection in a male population attending a sexually transmitted infection service. PLoS One 2013; 8:e54375.

21. Wang $H$, Li H, Hu L, Zhou J et al. EGFR and KRAS mutations in Chinese patients with sinonasal inverted papilloma and oncocytic papilloma. Histopathology 2019; 75:274281. 
22. Udager AM, McHugh JB, Goudsmit CM et al. Human papillomavirus (HPV) and somatic EGFR mutations are essential, mutually exclusive oncogenic mechanisms for inverted sinonasal papillomas and associated sinonasal squamous cell carcinomas. Ann Oncol 2018; 29:466-471.

23. Vyse $\mathrm{S}$, Huang PH. Targeting EGFR exon 20 insertion mutations in non-small cell lung cancer. Signal Transduct Target Ther 2019; $4: 5$

24. Cho J, Kim S, Du J, Meyerson M Autophosphorylation of the carboxyl-terminal domain is not required for oncogenic transformation by lung-cancer derived EGFR mutants. Int J Cancer. 2018; 143:679685.

25. Takahashi Y, Bell D, Agarwal G et al. Comprehensive assessment of prognostic markers for sinonasal squamous cell carcinoma. Head Neck 2014; 36:1094-1102.

26. Soria JC, Ohe Y, Vansteenkiste J et al. Osimertinib in Untreated EGFR -Mutated Advanced Non-Small-Cell Lung Cancer. N
Engl J Med 2017; 378:113-125

27. Robichaux JP, Elamin YY, Tan $Z$ et al Mechanisms and clinical activity of an EGFR and HER2 exon 20-selective kinase inhibitor in non-small cell lung cancer. Nat Med 2018; 24:638-646

28. López F, Garcia Inclan C, Perez-Escuredo J et al. KRAS and BRAF mutations in sinonasal cancer. Oral Oncol 2012; 48:692-697.

29. Bornholdt J, Hansen J, Steiniche T et al. K-ras mutations in sinonasal cancers in relation to wood dust exposure. BMC Cancer 2008; 8:53

Mario A. Hermsen

Grupo Oncología de Cabeza y Cuello Instituto de Investigación Sanitaria del Principado de Asturias (ISPA) Instituto Universitario de Oncología del Principado de Asturias (IUOPA) Centro de Investigación Biomédica en Red (CIBER-ONC)

Edf. FINBA, N-1 F49

C/ Avenida de Roma s/n, 33011

Oviedo

Spain

Tel: +34985107937

E-mail: mhermsen@hca.es

ORCID no 0000-0002-5959-6289

This paper contains supplementary materials online: at www.rhinologyjournal.org 


\section{SUPPLEMENTARY MATERIAL}

Supplementary Table 1. Primers used for PCR Sanger sequencing of EGFR and KRAS and HPV genotyping.

\begin{tabular}{|c|c|}
\hline Primers & Sequence $5^{\prime}$ to $3^{\prime}$ \\
\hline KRAS Exon $2 \mathrm{Fw}$ & TACTGGTGGAGTATTTGATAGTG \\
\hline KRAS Exon 2 Rv & CTGTATCAAAGAATGGTCCTG \\
\hline EGFR Exon $20 \mathrm{Fw}$ & CCCTGTGCTAGGTCTTTTG \\
\hline EGFR Exon $20 \mathrm{Rv}$ & GTCTTTGTGTTCCCGGACAT \\
\hline \multirow{15}{*}{ HPV type-specific primers } & HPV-6 (MY12: 5'CATCCGTAACTACATCTTCCA-3') \\
\hline & HPV-11 (MY13: 5'-TCTGTGTCTAAATCTGCTACA3') \\
\hline & HPV-16 (MY14: 5'-CATACACCTCCAGCACCTAA-3') \\
\hline & HPV-18 (WD74: 5'GGATGCTGCACCGGCTGA-3') \\
\hline & HPV-31 (WDB128: 5'TTGCAAACAGTGATACTACATT-3') \\
\hline & HPV-33 (MY16: 5'CACACAAGTAACTAGTGACAG-3') \\
\hline & HPV-35 (5'-CTGCTGTGTCTTCTAGTGA-3') \\
\hline & HPV-39 (5'ATAGAGTCTTCCATACCTTC-3') \\
\hline & HPV-45 (MYB69: 5'ATACTACACCTCCAGAAAAGC-3') \\
\hline & HPV-51 (5'-TGCTGCGGTTTCCCCAA-3') \\
\hline & HPV-52 (5'-GAATACCTTCGTCATGGC-3') \\
\hline & HPV-56 (5'-TGTCTACATATAATTCAAAGC-3') \\
\hline & HPV-58 (SANTI58: 5'TGAAGTAACTAAGGAAGGTACA-3') \\
\hline & HPV-66 (5'-AGCTAAAAGCACATTAACTAA-3') \\
\hline & HPV-68 (5'CTGAATCAGCTGTACCAAT-3') \\
\hline
\end{tabular}

Supplementary Table 2. Frequencies of EGFR mutation, protein expression and gene copy number, and HPV infection and KRAS mutation, according to type of tumour sample.

\begin{tabular}{|c|c|c|c|}
\hline Analysis & 55 ISP & 14 SNSCC-isp & 60 SNSCC-novo \\
\hline EGFR mutation & $7 / 18$ (39\%) & $6 / 12(50 \%)$ & $1 / 19(5 \%)$ \\
\hline IHC-EGFR positive & $34 / 37$ (92\%) & $6 / 10(60 \%)$ & $24 / 54$ (44\%) \\
\hline IHC-pEGFR positive & $21 / 39(54 \%)$ & 4/12 (33\%) & $20 / 52$ (38\%) \\
\hline MLPA-EGFR gain & $1 / 17(6 \%)$ & $1 / 5(20 \%)$ & 6/32 (19\%) \\
\hline HPV 16 positive & $4 / 40(10 \%)$ & $2 / 13(15 \%)$ & $1 / 50(2 \%)$ \\
\hline HPV 18 positive & $0 / 40(0 \%)$ & $1 / 13(8 \%)$ & $0 / 50(0 \%)$ \\
\hline HPV 42 positive & $0 / 40(0 \%)$ & $1 / 13(8 \%)$ & $0 / 50(0 \%)$ \\
\hline HPV 61 positive & $0 / 40(0 \%)$ & $1 / 13(8 \%)$ & $0 / 50(0 \%)$ \\
\hline KRAS mutation & $0 / 28(0 \%)$ & $0 / 9(0 \%)$ & $0 / 46(0 \%)$ \\
\hline
\end{tabular}



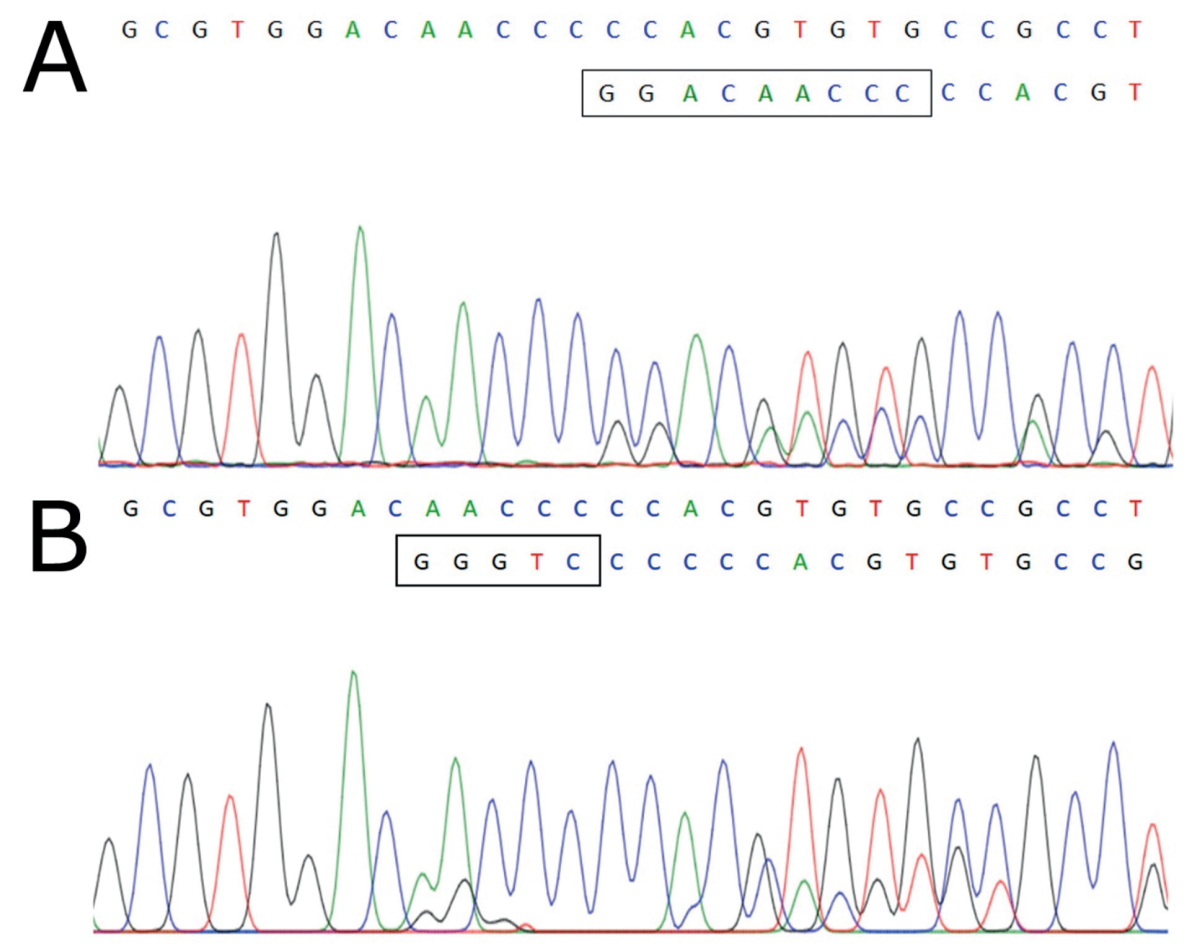

Supplementary Figure 1. Two new EGFR exon 20 mutations not described before in the literature. A) In-frame 9 bp insertion: p.P772_H773dupPDN c.2315_2323dupGGACAACCC detected in a SNSCC-novo; B) In-frame deletion-insertion: p.N771delinsGS c.2310_2311 AA>GGGTC detected in an ISP.
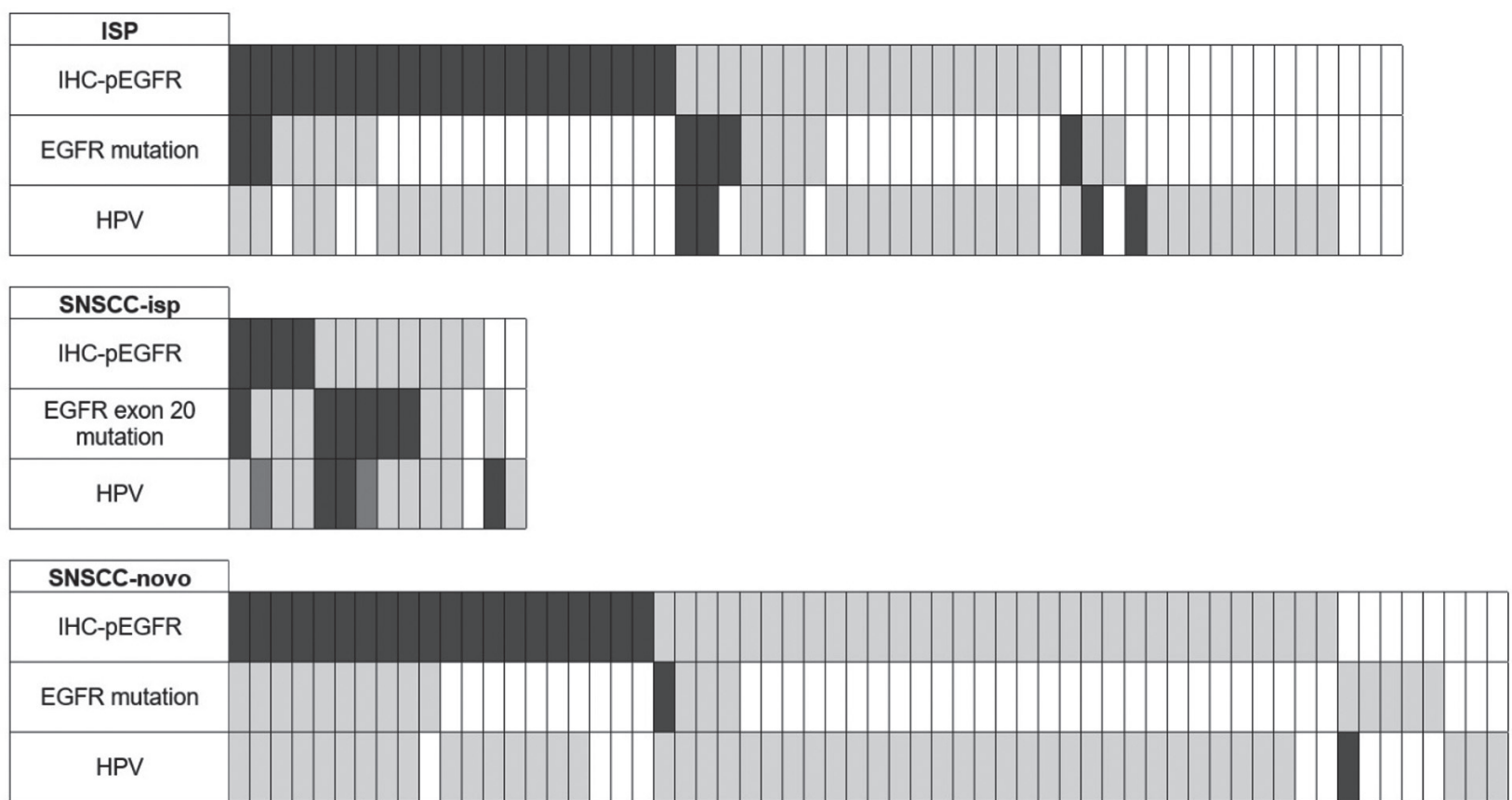

Supplementary Figure 2. Case-by-case representation of pEGFR expression, EGFR mutation and HPV infection in 55 ISP, 14 SNSCC-isp and 60 SNSCCnovo. Dark bars indicate presence and grey bars absence of pEGFR-positivity, EGFR mutation or HPV-infection; white bars represent cases that could not be analysed. Low-risk HPV infection (two cases SNSCC-isp) are indicated by dark-grey bars. Activated pEGFR expression and EGFR mutation occur mostly mutually exclusive, however, in contrast to previous publications, EGFR mutation and oncogenic HPV do co-occur in this cohort. 